\title{
SAMU: Design and Implementation of Selectivity-Aware MU-MIMO for Wideband WiFi
}

\author{
Yongjiu Du ${ }^{1}$, Ehsan Aryafar ${ }^{2}$, Pengfei Cui ${ }^{1}$, Joseph Camp $^{1}$, and Mung Chiang ${ }^{3}$ \\ ${ }^{1}$ EE Department, Southern Methodist University, Dallas, TX, USA \\ ${ }^{2}$ Intel Labs, Santa Clara, CA, USA \\ ${ }^{3}$ EE Department, Princeton University, Princeton, NJ, USA
}

\begin{abstract}
In anticipation of the increasing demand of wireless traffic, WiFi standardization efforts have recently focused on two key technologies for capacity improvement: multi-user MIMO and wider bandwidth. However, users experience heterogeneous channel orthogonality characteristics across sub-carriers in the same channel bandwidth, which prevents ideal multi-user gain. Moreover, frequency selectivity increases as bandwidth scales and correspondingly severely deteriorates multi-user MIMO performance. In this work, we consider the frequency selectivity of current and emerging WiFi channel bandwidths to optimize multi-user MIMO by dividing the occupied channel bandwidth into equally-sized sub-channels according to the level of frequency selectivity. In our selectivity-aware multi-user MIMO design, SAMU, each sub-channel is allocated according to the largest bandwidth that can be considered frequency-flat, and an optimal subset of users is chosen to serve in each sub-channel according to spatial orthogonality, achieving a significant performance improvement for all users in the network. Additionally, we propose a selectivity-aware very high throughput (SA-VHT) mode, which is based on and an extension to the existing IEEE 802.11ac standard. Over emulated and real indoor channels, even with minimal mobility, SAMU achieves as much as 80 percent throughput improvement compared to existing multi-user MIMO schemes, which could serve as a lower bound as bandwidth scales.
\end{abstract}

\section{INTRODUCTION}

To address the exponentially increasing traffic demand in wireless networks, IEEE 802.11 standardization efforts have focused on two critical improvements for increasing system capacity. First, multiple antennas on the transmitter and/or receiver have been leveraged for MIMO transmission. More recently, multi-user MIMO (MU-MIMO) has also been studied [1]-[5] and applied in a number of wireless standards [6][8]. Second, WiFi standards have recently sought to increase the total bandwidth per channel access. For example, 802.11n uses up to $40 \mathrm{MHz}, 802.11 \mathrm{ac}$ uses up to $160 \mathrm{MHz}$, and 802.11ad will use multiple $\mathrm{GHz}$ of available bandwidth at a 60-GHz carrier frequency. Thus, the signal bandwidth is only expected to increase for emerging WiFi networks.

Even for typical environmental settings for WiFi networks which seem relatively simple, reflections off of surrounding objects cause strong multi-path effects from sender to receiver. As a result, frequency-selective fading occurs across the channel bandwidth, where carrier frequencies experience diverse received signal levels. For systems with much wider bandwidth (e.g., at least $20 \mathrm{MHz}$ ), frequency-selective fading can become a dominant channel effect, degrading the system performance.
Frequency selectivity ${ }^{1}$ becomes an even more critical issue for MU-MIMO systems, severely degrading the high throughput previously anticipated [9]-[12].

In MU-MIMO systems, each user can correctly decode packets simultaneously due to spatial diversity and pre-coding of channel weights by the transmitter. The total throughput, however, highly depends on the relationship between the channel responses of all users. The access point attempts to select an optimal subset of users that maximizes the overall spatial efficiency. Optimal user selection is usually based on the channel characteristics experienced by and the traffic requirement of all the users. However, with frequency selectivity, a subset of users may have good spatial reuse on some carrier frequencies due to the orthogonality of the sub-channels experienced by each user, but poor orthogonality on other carrier frequencies due to the frequency diversity across the channel bandwidth. Hence, the two most critical enhancements to the 802.11 standard are known to independently improve the system capacity, but the capacity achievable via their joint use in the field is an open question. There is currently no such scheme to adapt the bandwidth of MU-MIMO WiFi according to the frequency selectivity of the channel.

In this work, we consider the frequency selectivity to allocate the maximum width appropriate for frequency-flat sub-channels. In doing so, the channel response per subchannel can be regarded as homogeneous for all frequencies. Since each sub-channel has different frequency characteristics, we choose a corresponding optimal subset of users for each sub-channel. The subset of users across different sub-channels could be the same, partially overlapped, or mutually exclusive, depending on the channel characteristics of all users and the degree to which frequency selectivity occurs. Hence, our selectivity-aware multi-user MIMO scheme, SAMU, overcomes a key issue in wideband WiFi networks: frequency selectivity. Moreover, we propose a selectivity-aware very high throughput (SA-VHT) mode, which is based on and an extension to the existing 802.11 ac systems. We experimentally evaluate SAMU over emulated channels for repeatability and control as well as in the field for representative frequencyselective scenarios. In a wide range of system configurations across emulated and real indoor channels, we show that SAMU can significantly improve the system throughput by as much as 80 percent.

The main contributions of this paper are as follows:

\footnotetext{
${ }^{1}$ In this paper, the term selectivity refers to frequency selectivity.
} 
1) We measure and evaluate the channel response characteristics and channel orthogonality for multi-user MIMO scenarios in indoor environments, showing that frequency selectivity not only applies to a single link, but also affects the user selections for multi-user MIMO.

2) We develop protocols and algorithms for a selectivityaware multi-user MIMO scheme, SAMU, which considers the degree to which frequency selectivity is present on the channel, allocates frequency-flat sub-channels, and chooses an optimal subset of users for each subchannel to improve the spatial efficiency of the network.

3) We present the SAMU design which spans physical (PHY) and media access control (MAC) layer modifications to support sub-channel and user selection. Specifically, we outline a selectivity-aware very high throughput (SA-VHT) mode as an extension to the IEEE 802.11ac standard.

4) We evaluate a sub-channel division adaptation scheme to dynamically change the number of sub-channels according to the delay spread in environment of operation.

5) We conduct experiments with programmable hardware devices over repeatable and realistic channel conditions on a channel emulator and in a representative environment, showing significant throughput improvement over the existing IEEE 802.11ac standard.

The rest of the paper is organized as follows. Section II describes the related work. The impact of selectivity on MUMIMO performance is described in Section III. Thereafter, we present the design of SAMU (Section IV), evaluate its performance (Section V), and conclude (Section VI).

\section{RELATED WORK}

Multi-user MIMO: MIMO technology has been extensively developed and applied to both access points and client devices to improve the system capacity [4]. MIMO also enables multi-user communications to further improve the system throughput. Multi-user beamforming can achieve additional throughput improvement by properly setting the steering matrix of the transmitting links to enable parallel data transmissions [1]-[3]. Interference nulling and cancellation allow a receiver to decode multiple parallel streams from different transmitters [9], [10]. These works either use narrow-band signals or are evaluated in wireless channels with minimal frequency selectivity. In contrast, we show that multi-user systems in an indoor environment can be significantly affected by frequency selectivity.

Frequency Diversity Measurements in Indoor Environments: In order to achieve high levels of capacity and reliability in wireless networks, measuring the wireless channel is critical for designing and implementing effective systems. Channel measurements have been done in different environments and frequency bands [13]-[18]. In [13], the authors measure the channel heterogeneity and temporal stability to guide their system design. In [14], the authors investigated the temporal, spatial, and spectral fading in home and office environments on the $5.2 \mathrm{GHz}$ band. Power-delay profile characteristics are measured in [16]. In [15], [18], channel characteristics of millimeter waves are measured to study the feasibility of next generation wireless networks in that band.
In contrast to these single antenna works, we study the impact of frequency diversity on multi-user MIMO scenarios.

OFDM: OFDM is a prevalent scheme for wideband communication across wired/wireless mediums and used in various applications [6], [7], [19], [20]. The primary advantage of OFDM is its robustness to severe fading conditions without complex equalization filters. Another major advantage of OFDM is to allow the devices to easily manipulate the signals on different sub-carriers and allow sub-channels to be apportioned according to the frequency selectivity [8]. In [8], the OFDM sub-carriers are grouped to Physical Resource Blocks (PRBs), and the AP allocates different PRBs to different users, which provides significant flexibility in allocating bandwidth to users according to their respective demands. In contrast, we demonstrate the feasibility of user selection on different sub-carriers of an OFDM system.

Frequency-aware Wireless Communication: For either indoor or outdoor environments in urban areas, significant frequency diversity has been observed. Traditional wireless protocols use the same settings on all of the frequencies or sub-carriers across the entire channel bandwidth, regardless of the channel gain diversity. Applying different configurations on sub-carriers according to their respective channel gains can improve the system performance. Several prior works have developed systems dealing with frequency diversity [13], [21][23]. In [21], the author measured per sub-carrier SNR to enable the transmitter to adapt the bit rate independently across different sub-carriers. The authors in [13] offer proportional protection of symbols according to their channel quality and importance level. In [22], an effective algorithm was developed to select the appropriate spectrum to use for each transmission by taking into account both frequency diversity and interference. In [23], a system was developed to redistribute the transmit power across the sub-carriers to better cope with the frequency selectivity, subject to a fixed power budget constraint. In 3GPP-LTE, frequency-aware user scheduling and resource allocation have also been shown to provide significant performance improvement [24]. In contrast, we consider both spatial and spectral dimensions for user selection in a MUMIMO scenario and perform sub-channel allocation according to the level of frequency selectivity to improve MU-MIMO performance. As a result, the above-mentioned related work (e.g., [13], [23], [24]) can be applied in our proposed SAMU to obtain an aggregate performance improvement. In this paper, we focus on showing the performance improvement without adopting further possible enhancements.

\section{IMPACT OF FREQUENCY SELECTIVITY ON MU-MIMO PERFORMANCE}

We start by quantifying the level of frequency selectivity in common indoor environment and consider its impacts on MU-MIMO performance.

\section{A. Frequency Diversity in Indoor Environments}

In a real environment where mobile communication occurs, the presence of reflecting/refracting obstacles can cause a large number of paths that signals traverse. These paths can be abstracted into groups based on their attenuation and delay from the first-arriving signal. Each group is referred to as a 


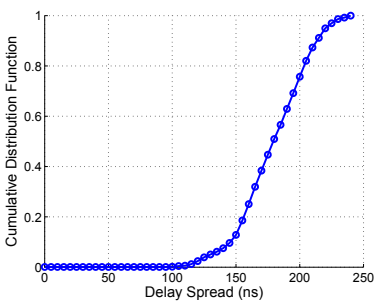

(a)

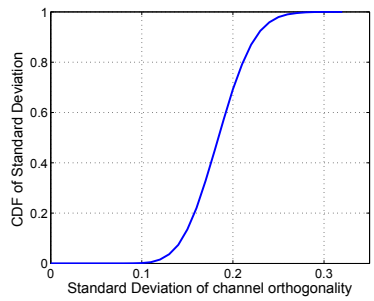

(c)

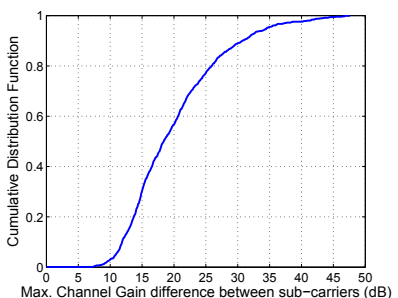

(b)

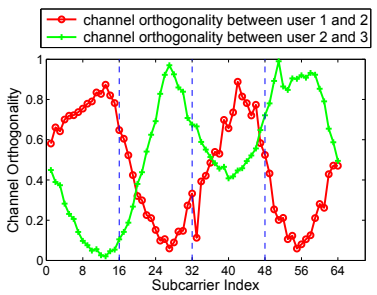

(d)
Fig. 1: Frequency selectivity evaluation in an office environment. (a) Measured CDF of delay spread. (b) Maximum measured channel gain difference across all sub-carriers. (c) Measured channel orthogonality on different subcarriers between 2 users. (d) Demonstration of selectivityaware channel division.

channel tap. The channel taps are then aggregated into a single channel model, which represents a given environment with each tap having a fading characterization based on a certain probability distribution (e.g., Rayleigh or Rician).

Delay spread is a measure of multi-path richness of a communication channel, which can be interpreted as the difference between the arrival time of the earliest significant multi-path component and the arrival time of the latest multipath component [25]. A standard metric to quantify delay spread is the root mean square (RMS) delay spread. Let $P(\tau)$ denote the power-delay profile of the channel. Then, the RMS delay of the channel is:

$$
\tau_{r m s}=\sqrt{\frac{\int_{0}^{\infty}(\tau-\bar{\tau})^{2} P(\tau) d \tau}{\int_{0}^{\infty} P(\tau) d \tau}}
$$

Here, $\bar{\tau}$ is the mean delay spread of the channel and expressed as:

$$
\bar{\tau}=\frac{\int_{0}^{\infty} \tau P(\tau) d \tau}{\int_{0}^{\infty} P(\tau) d \tau}
$$

We conduct experiments to measure delay spread and channel characteristics of an office environment using WARPLab [26]. Our experiments are conducted on the 5.2$\mathrm{GHz}$ band and over a $20-\mathrm{MHz}$ bandwidth.

Figure 1a depicts the CDF of the measured delay spread. We observe that the delay spread is approximately between 50 and 300 nanoseconds, corresponding to a difference in path lengths that the multi-path signals traverse of approximately 15 to 90 meters. This delay spread level will cause severe frequency selectivity even with a channel bandwidth of $20 \mathrm{MHz}$.
Due to the multi-path components in the environment, signals experience different levels of channel gain on different frequencies. Figure $1 \mathrm{~b}$ depicts the measured channel gain difference across all sub-carriers for multiple measurements. For each measurement, the maximum channel gain difference means the difference between the maximum channel gain and the minimum channel gain. We can observe that, over different sub-carriers, the channel gain can vary as much as $50 \mathrm{~dB}$, while the average gain deviation is around $30 \mathrm{~dB}$.

\section{B. MIMO Channel Orthogonality}

Channel orthogonality is a metric to evaluate the potential spatial efficiency of multi-user MIMO systems, which can be measured by the following equation:

$$
\alpha_{i, j, k}=1-\frac{\left|\boldsymbol{h}_{i, k} \cdot \boldsymbol{h}_{j, k}^{*}\right|}{\left\|\boldsymbol{h}_{i, k}\right\| \cdot\left\|\boldsymbol{h}_{j, k}\right\|}
$$

For $\alpha_{i, j, k}$, the vectors $\boldsymbol{h}_{i, k}$ and $\boldsymbol{h}_{j, k}$ are channel responses from the access point (AP) to user $i$ and user $j$ on sub-carrier $k$, respectively. $\alpha_{i, j, k}$ has a range of $(0,1)$. For $\alpha_{i, j, k}$, a value of 0 denotes that the two channel vectors are fully parallel, while a value of 1 denotes that the two channel vectors are fully orthogonal. The more orthogonal the channel vectors, the less the signal from one user interferes with the signal of the other user. Hence, better spatial efficiency can be achieved to improve the throughput.

For different users, if their channel vectors to the AP are orthogonal, optimal multi-user gain can be achieved. However, in practice, it is hard to find a set of users whose channel vectors are fully orthogonal, especially for a small number of users. An exhaustive search over the entire user set can be used to find the optimal selected simultaneous user set from a small number of total users [27]. For a large number of users, semi-orthogonal user selection provides a sub-optimal performance with lower computational complexity [2], [3].

In existing MU-MIMO schemes, each user occupies the entire channel bandwidth. However, with frequency-selectivity, a subset of users may achieve higher spatial efficiency for some sub-carriers due to the orthogonality of the corresponding subchannels. However, the same scheme may not be optimal for other sub-carriers due to the frequency diversity changing the sub-channel orthogonality. The problem is only exacerbated with wider bandwidths.

We conduct experiments to quantify the level of channel orthogonality in indoor environments. We use one WARP [26] board as the AP and two other WARP boards as users. The AP is equipped with 4 antennas, while each user has only one antenna. We set the number of sub-carriers to 64 . We show the measured channel orthogonality between the two users on different sub-carriers in Figure 1c. We can see that, due to frequency-selective fading, the channel orthogonality of the users fluctuate significantly across different sub-carriers.

\section{Selectivity-Aware User Selection}

In existing MU-MIMO WiFi systems, the AP selects the optimal group of users based on an average expected performance across the entire channel. However, as we have shown experimentally, in a fixed topology with only $20 \mathrm{MHz}$ 
bandwidth, multi-path effects cause frequency selectivity in most wireless scenarios. As a result, the selected subset of users could be optimal for part of the channel bandwidth, but not optimal for other parts of the channel bandwidth. Therefore, we propose SAMU, a system that divides the given bandwidth into sub-channels equally and selects the optimal set of users for each sub-channel. The number of these sub-channels, $n$, depends on the bandwidth, the frequency selectivity characteristics of the environment across the given bandwidth (the maximum delay spread), and the frame duration. The set of users across different sub-channels could be the same, partially overlap, or mutually exclusive.

For a given sub-carrier, different pairs of users experience different levels of channel orthogonality. Thus, with intelligent selection of users and channel partitioning, MU-MIMO performance can be improved significantly. We show an example of the channel division for two pairs of users on different subcarriers in Figure 1d, based on our indoor measurements. We can see that the channel orthogonality fluctuates among subcarriers. If we select the same sub-set of users across all subcarriers, multi-user efficiency will be low for the sub-carriers on which the users are less orthogonal. If the entire channel bandwidth is divided into 4 sub-channels, for sub-channel 1 , $2,3,4$, we select user group of $\{1,2\},\{2,3\},\{1,2\},\{2$, $3\}$, respectively. Then, there will be significant throughput improvement, and more generally as shown in Section V.

\section{DESIGN OF SAMU}

In this section, we describe the design of our selectivityaware MU-MIMO system, SAMU. In particular, SAMU consists of: (i) a PHY layer design that supports sub-channel encoding, (ii) a MAC layer design to optimally select users in a given sub-channel, (iii) an ACK management mechanism for multiple data packets across the channel bandwidth, and (iv) an adaptation mechanism for sub-channel division according to diverse delay spreads across environments.

\section{A. Physical Layer Signaling}

Figure 2a shows the VHT mode data frame format in the IEEE 802.11ac standard. VHT-SIG-A is VHT-mode specific, which is used to convey information of the VHT configurations, including the bandwidth, user selection, and spacetime coding scheme. VHT-SIG-B is user specific and contains the packet size, modulation scheme, and coding level for the packet of the specified user among all simultaneously-selected users. The functionality description of other fields can be found in [7]. VHT-SIG-A is composed of two symbols, VHTSIG-A1 and VHT-SIG-A2 (as depicted in Figure 3) [7]. All the information shown in Figure 3 are required for the receiver to interpret the VHT transmission configuration.

The VHT-SIG-A field is designed for multiple users. However, in our selectivity-aware multi-user scheme, since we divide the entire wireless channel into multiple sub-channels, the signal of a user may occupy different numbers of sub-channels depending on the channel characteristics of the simultaneous users. For a selected user, to correctly decode the signals, the index of every sub-channel that carries its data packets must be known. Moreover, the resulting signal-to-interference-plusnoise ratio (SINR) depends on each sub-channel. As a result,

\begin{tabular}{|c|c|c|c|c|c|c|c|}
\hline L-STF & L-LTF & $\begin{array}{c}\text { L- } \\
\text { SIG }\end{array}$ & $\begin{array}{c}\text { VHT- } \\
\text { SIG-A }\end{array}$ & $\begin{array}{c}\text { VHT- } \\
\text { STF }\end{array}$ & VHT-LTF & $\begin{array}{c}\text { VHT- } \\
\text { SIG-B }\end{array}$ & Data \\
\hline
\end{tabular}

(a)

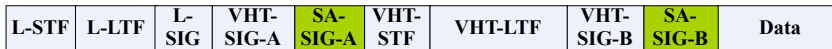

(b)

Fig. 2: Frame format: (a) VHT mode in IEEE 802.11ac. (b) the proposed SA-VHT mode.

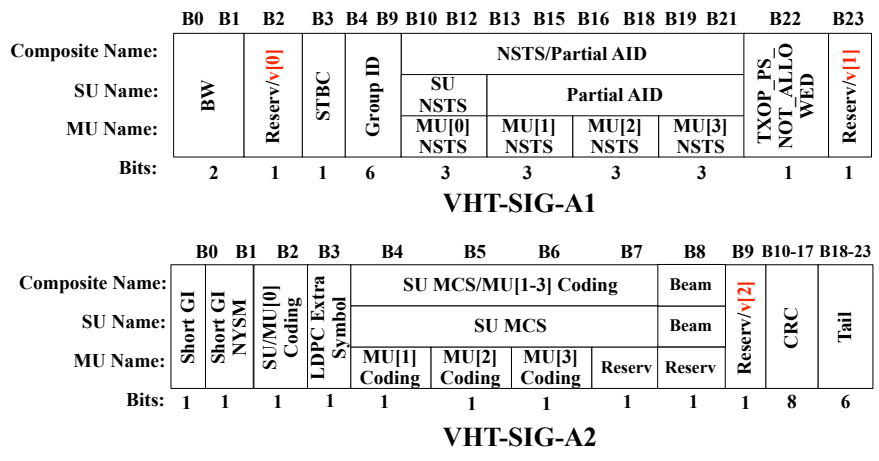

Fig. 3: VHT-SIG field in IEEE 802.11ac.

different modulation and coding schemes (MCSs) should vary across sub-channels, even for the same user. Hence, MCS information must also be conveyed to each intended receiver, and we correspondingly insert additional signaling fields in the frame to carry this information. In order to make our proposed scheme align with the existing 802.11ac standards, we use 3 of the reserved bits (shown in a red font in Figure 3) in the VHTSIG-A fields. These bits inform the receiver whether the packet uses selectivity-aware mode and how many sub-channels are in use. The configuration of the bandwidth division is shown in Table I. The number of resulting sub-channels is denoted as $N_{s u b} . N_{s u b}$ is OFDM protocol dependent, and the potential range of $N_{s u b}$ could be from 1 to the total number of OFDM subcarriers, with $N_{s u b}=1$ meaning each sub-carrier is a subchannel. The number of additional SA-SIG-A and SA-SIG-B symbols is $N_{a}$ and $N_{b}$, respectively. For each bandwidth, a corresponding number of sub-carriers are used in the OFDM scheme. $N_{p k t-b i t}$ is the number of bits used to indicate the packet size on each sub-channel for each user. When the AP uses the existing IEEE 802.11ac scheme for transmission, the selectivity-aware configuration is set to a binary value $v=000$. A non-zero selectivity-aware configuration denotes that the AP will divide the channel into $2^{v}$ sub-channels for transmission. In this mode, there are additional selectivityaware signal-A (SA-SIG-A) symbols appended after VHTSIG-A and SA-SIG-B symbols appended after VHT-SIG-B to inform the receiver of the various configurations on each sub-channel. The frame format of the SA-VHT mode is shown in Figure 2b.

For each sub-channel, a separate Group ID, NSTS, Coding Type, MCS value and packet size are needed for the receivers to correctly decode the packet. The Group ID, NSTS, and Coding Type are carried in the VHT-SIG-A and SA-SIG-A fields. The number of SA-SIG-A and SA-SIG-B symbols for each channel bandwidth and channel division is shown in 


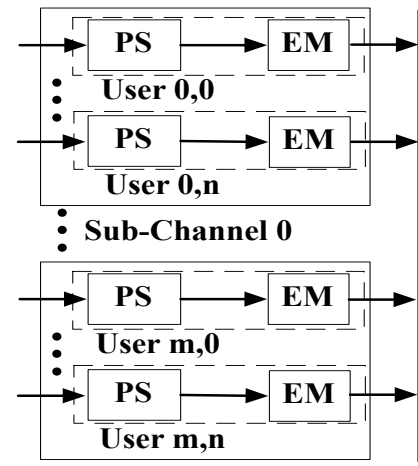

Sub-Channel m
PS: Padding \& Scrambler EM: Encoding \& Modulation

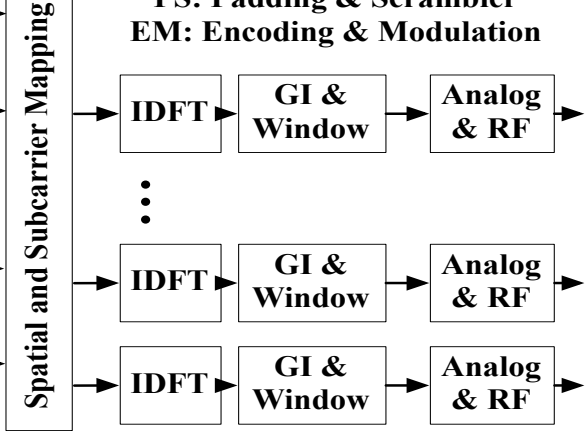

(a)

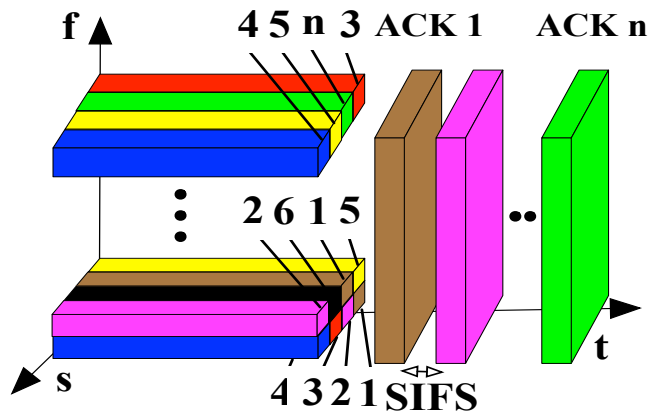

(b)

Fig. 4: SAMU MAC and PHY design: (a) Transmitter block diagram of SA-VHT mode, and (b) Transmission and Acknowledgement in SA-VHT.

TABLE I: Selectivity-Aware Configuration

\begin{tabular}{|c|c|c|c|c|c|c|}
\hline bandwidth & $\mathrm{N}_{\text {base }}$ & $v$ & $\mathbf{N}_{\text {sub }}$ & $\mathbf{N}_{p k t-b i t}$ & $\mathrm{~N}_{a}$ & $\mathrm{~N}_{b}$ \\
\hline \multirow{6}{*}{$20 \mathrm{MHz}$} & \multirow{6}{*}{26} & 000 & 1 & 16 & 0 & 0 \\
\hline & & 001 & 2 & 15 & 2 & 1 \\
\hline & & 010 & 4 & 14 & 3 & 3 \\
\hline & & 011 & 8 & 13 & 7 & 5 \\
\hline & & 100 & 16 & 12 & 14 & 10 \\
\hline & & 101 & 32 & 11 & 29 & 19 \\
\hline \multirow{6}{*}{$40 \mathrm{MHz}$} & \multirow{6}{*}{54} & 000 & 1 & 17 & 0 & 0 \\
\hline & & 001 & 2 & 16 & 2 & 1 \\
\hline & & 010 & 4 & 15 & 3 & 2 \\
\hline & & 011 & 8 & 14 & 7 & 3 \\
\hline & & 100 & 16 & 13 & 14 & 5 \\
\hline & & 101 & 32 & 12 & 29 & 10 \\
\hline \multirow{6}{*}{$80 \mathrm{MHz}$} & \multirow{6}{*}{117} & 000 & 1 & 19 & 0 & 0 \\
\hline & & 001 & 2 & 18 & 2 & 1 \\
\hline & & 010 & 4 & 17 & 3 & 1 \\
\hline & & 011 & 8 & 16 & 7 & 2 \\
\hline & & 100 & 16 & 15 & 14 & 3 \\
\hline & & 101 & 32 & 14 & 29 & 5 \\
\hline \multirow{6}{*}{$160 \mathrm{MHz}$} & \multirow{6}{*}{234} & 000 & 1 & 19 & 0 & 0 \\
\hline & & 001 & 2 & 18 & 2 & 1 \\
\hline & & 010 & 4 & 17 & 3 & 1 \\
\hline & & 011 & 8 & 16 & 7 & 1 \\
\hline & & 100 & 16 & 15 & 14 & 2 \\
\hline & & 101 & 32 & 14 & 29 & 3 \\
\hline
\end{tabular}

Table I. Since the SA-SIG-A field uses the $802.11 \mathrm{a} / \mathrm{g}$ base rate (24 bits per symbol) to transmit, the number of symbols in the SA-SIG-A field, $\mathrm{N}_{a}$, can be calculated as follows:

$$
\mathbf{N}_{a}= \begin{cases}0 & \text { if } \mathbf{N}_{\text {sub }}=1 \\ \left\lceil\frac{\left(\mathbf{N}_{s u b}-1\right) * 22+6}{24}\right\rceil & \text { otherwise }\end{cases}
$$

In each OFDM symbol with the base transmission mode (BPSK with a code rate of 1/2), 26, 54, 117, and 234 coded bits can be carried with the bandwidth of 20,40, 80, and 160 $\mathrm{MHz}$, respectively. As a result, the number of SA-SIG-B field symbols, $\mathrm{N}_{b}$, can be calculated as follows:

$$
\mathbf{N}_{b}= \begin{cases}0 & \text { if } \mathbf{N}_{\text {sub }}=1 \\ \left\lceil\frac{\left(\mathbf{N}_{\text {sub }}-1\right) *\left(\mathbf{N}_{\text {pkt-bit }}+4\right)+6}{\mathbf{N}_{\text {base }}}\right\rceil & \text { otherwise }\end{cases}
$$

Compared to the existing IEEE 802.11ac standard, the SA-SIG-A and SA-SIG-B fields introduce different levels of overhead proportional to the channel bandwidth and channel division level. The throughput efficiency with the overhead can be calculated as follows:

$$
\xi=\frac{T_{f}-T_{\text {header }}-\left(N_{a}+N_{b}\right) * T_{s}}{T_{f}-T_{\text {header }}}
$$

Here, $T_{f}$ is the frame duration with a maximum value of $5460 \mu \mathrm{s}$, as specified in [7]. Theader is the time duration, including the preambles and signaling symbols in the frame. $T_{s}$ is a symbol duration with a value of $4 \mu \mathrm{s}$. From the experimental evaluation in the following section, we will see that the improvement provided by channel division is much higher than the overhead introduced by channel division.

\section{B. Physical Layer Modulation}

For the SA-VHT scheme, since we divide the entire channel into sub-channels with the same frame duration, the packet size transmitted on one sub-channel is smaller than the existing IEEE 802.11ac VHT mode. The AP first divides the packet for a served user into smaller packets which will fit into one subchannel. Then, the AP allocates one or more small packets for a user on one or more sub-channels with potentially different modulation and coding schemes. The AP first calculates the MCS for each user on each sub-channel according to the channel quality and orthogonality. For each sub-channel, the AP allocates a certain amount of information bits to make sure each sub-channel has the same number of valid data symbols. The PHY layer transmitter block diagram of the SA-VHT mode is shown in Figure 4a. Currently, an IEEE 802.11ac AP modulates the data for each served user and then maps the data onto the analog and RF chains. In the proposed SA-VHT scheme, there is an additional sub-channel dimension. The AP needs to modulate the data for each user on each sub-channel, and then maps the resulted data into spatial and sub-channel streams. While SA-VHT has a slightly higher complexity, modern wireless transceivers typically use a combination of an embedded CPU and ASIC which can both be multiplexed over time. 


\section{Media Access Control Layer Design}

The MAC layer of SA-VHT mode mostly adopts the MAC protocol for the VHT mode of the 802.11ac standard with some modifications. Since the entire channel is divided into multiple sub-channels, and a separate sub-set of users is served on each sub-channel. One AP may support a larger number of simultaneous users than the current number supported by 802.11ac APs.

According to the reported channel characteristics from all the authorized users via sounding packets, the AP selects the optimal subset of users for each sub-channel. Priority from the application layers could also be considered during user selection. However, in our design and experiment, we choose to use the maximum total throughput metric to select the users. The process is depicted in Algorithm 1.

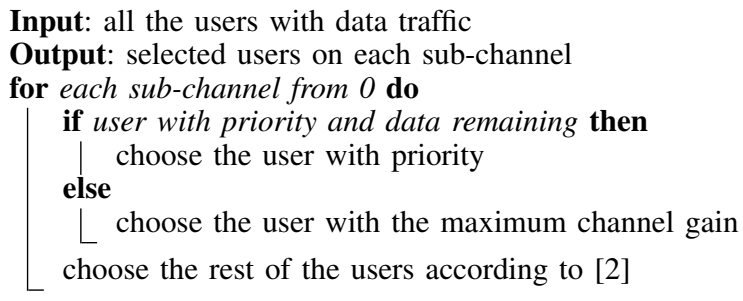

Algorithm 1: User Selection

Adopted from 802.11ac, each user is assigned a distinct user Group ID and User Position via the Group ID Management frame. As we described in the PHY layer design, there is information about the Group ID and User Position contained in the VHT-SIG-A and SA-SIG-A fields. Whenever the AP transmits packets to multiple users on multiple subchannels, each user receives the VHT-SIG-A and SA-SIG-A fields of the packets. With the information contained in the signaling symbols therein, the user knows the information of all the other simultaneous users, as well as the User Position information for each sub-channel. The receiver then looks up the stored User Group table and generates a list indicating the simultaneous users for this transmission. The users served with this transmission are then ordered from lower sub-channel to higher sub-channel and from lower position to higher position. The users of sub-channel 0 is at the front of the list, and the user with User Position 0 on sub-channel 0 is the first item in the list. Then, the receiver checks the next sub-channel. If the users on the current sub-channel already exist in the list, then it will not be included again. If there are new users that do not exist in the list, the receiver will append the the new users to the list. The receiver repeats this process until the last sub-channel is considered, as shown in Algorithm 2.

In Figure $4 \mathrm{~b}$, we depict an example explaining the ordering mechanism. On sub-channel 0 , a group of users $\{1,2,3,4\}$ are served. On sub-channel 1, a group of users $\{5,1,6,2\}$ are served. Then, for User 2, two lists are created. The first list is the user order list, which is $\{1,2,3,4,5,6\}$. The second list is the sub-channel list that carries packets for User 2. In this case, it is sub-channel 0 , sub-channel 1$\}$.

For each user, after decoding its PHY layer packets on one or more sub-channels according to the sub-channel list, it will recover the MAC layer packets by combining the PHY layer packets. When the receiver successfully receives the data packets, an ACK packet is required to be sent back to the AP. Otherwise, the AP will assume packet loss and a packet retransmission would be initiated in the following transmission. With the user order list, the users that have successful packet receptions will send the ACK packets one by one to notify the AP. The ACK packets include the user and sub-channel information, according to the order in the list. Since the ACK transmission time can be pre-calculated and followed by a DIFS slot, other users in the queue can virtually sense the total number of ACK frames.

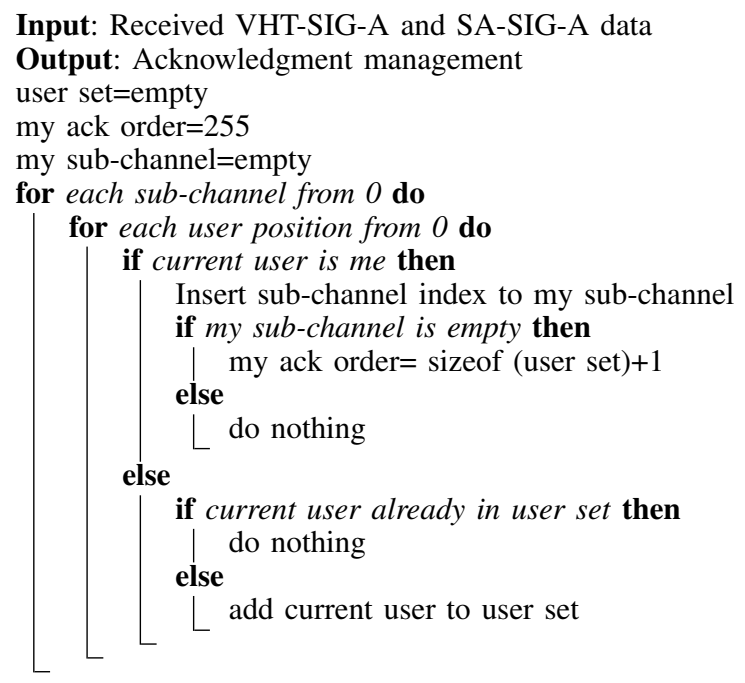

Algorithm 2: Distributed Acknowledgment Management

Sub-channel division will reduce the capacity of each sub-channel proportionally to the number of sub-channels. However, each user can occupy more than one sub-channel. By allocating the same user multiple sub-channels, SAMU can meet the data rate requirement of some particular users, while achieving significant gains in the aggregated throughput among all users. During user selection, the AP can mark some users as having higher priority. For each sub-channel, the user with highest priority and highest channel gain will be first selected. Then, the users with the best channel orthogonality will be selected accordingly.

The ACK packets from multiple users usually cause additional transmission overhead. However, this overhead can be reduced by two means. First, SAMU users send ACK packets back-to-back according to the user order list information in the PHY header, which removes the overhead of block ACK request packets in the legacy IEEE 802.11ac standard. Second, by partially overlapping the sets of users for different channels, the total number of users in one transmission can be minimized. Thus, the total number of back-to-back ACK packets can be minimized.

\section{Channel Division Adaptation}

Due to the mobility of the transmitter, receiver, or obstacles within the environment, channel fading is observed in the time domain, including variations of the number of taps, the amplitude of each tap, and the tap-delays from the firstarriving channel tap. In the frequency domain, the timevarying wireless channel is represented by amplitude and 


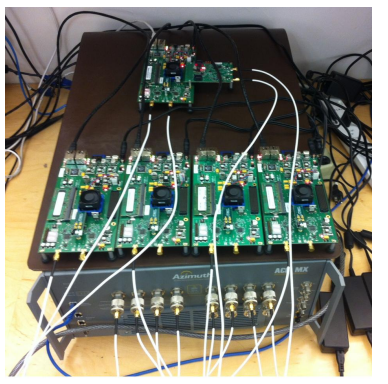

(a)

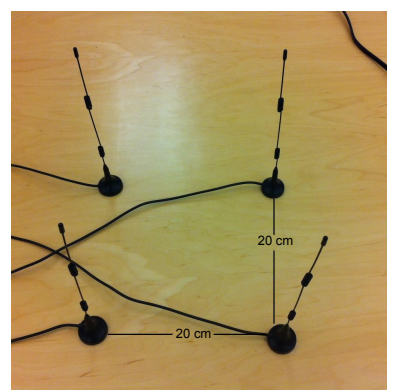

(b)
Fig. 5: Equipment settings for performance evaluation. (a) System Emulation using WARP and Azimuth. (b) Antenna placement for indoor test.

phase shifts on each sub-carrier, and the coherence bandwidth of the channel spectrum. When the coherence bandwidth is small, the signal will experience significantly different channel responses at different frequencies across a wideband channel. Therefore, to maintain orthogonality between users, we divide the entire bandwidth into more sub-channels. When the coherence bandwidth is large, we can divide the whole bandwidth into smaller number of sub-channels to reduce the overhead introduced by the system information carrying the channel division information. In order to optimize the system, we divide the channel into different number of sub-channels, adapting to the level of frequency selectivity.

\section{Evaluating SAMU on Diverse Channels}

We evaluate SAMU through a software-defined radio platform, WARPLab, in both emulated channels and a representative indoor environment.

\section{A. Experimental Setup}

WARPLab enables users to implement the PHY layer and MAC layer functionalities in Matlab and transmit/receive actual signals using RF radios [26]. In our experiments, one WARP board with four antennas acts as an access point, and the rest of the WARP boards act as client devices equipped with one antenna for each. We use an Azimuth channel emulator to investigate the factors that affect the system performance. The channel emulator can generate controllable and repeatable channels for complex wireless environments. The emulation system is shown in Figure 5a.

In addition to the emulator experiments, we also evaluate the system in an indoor environment. The floor plan of the office environment for our experiments is shown in Figure 6. We place the AP at the location marked with the red star and randomly place the users in the area marked with green ovals. The antennas of the AP form a square configuration shown in Figure 5b. The distance between antennas along each side of the square is $20 \mathrm{~cm}$, which is approximately the size of an AP device. Moreover, with the application of the $5.2-\mathrm{GHz}$ band, this antenna separation allows little correlation between the channels from different transmit antennas.

We run our experiments on a WiFi channel with a central frequency of $5180 \mathrm{MHz}$. The transmit signal bandwidth is 20

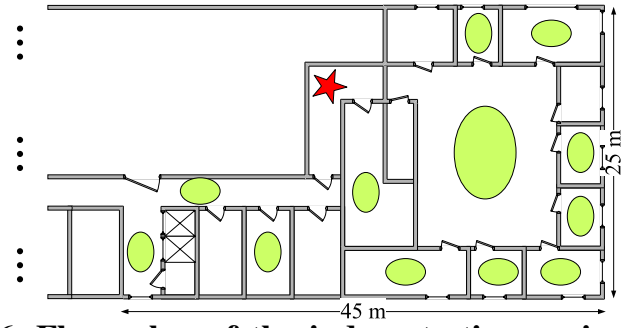

Fig. 6: Floor plan of the indoor testing environment.

$\mathrm{MHz}$, which is the maximum bandwidth currently supported by the WARP radio. We use an OFDM scheme with 64 subcarriers in our experiment, which is typical for 802.11 systems working with a $20-\mathrm{MHz}$ bandwidth.

For wireless systems, throughput is a typical metric of system performance. However, the throughput is usually closely related to the modulation and coding scheme (MCS). For a system with sparse MCS choices, in two scenarios with different channel qualities, the same MCS may be selected. As a result, the same throughput will be reported to the higher layers. Moreover, rate adaptation is usually used for wireless systems to adapt the transmission rate to the channel quality to improve the system throughput. However, due to the sparse MCS options and the performance of various rate adaptation schemes, the reported throughput can be diverse even for the same physical system design, preventing direct isolation of the potential system performance gain introduced by our selectivity-aware MU-MIMO scheme. Therefore, we adopt the system evaluation metric and method used in [11], which is to measure the received SNR and directly map the SNR to throughput according to (Equation 9 in [11]) :

$$
\text { Rate }=\sum_{i} \log _{2}\left(1+\mathrm{SNR}_{\text {measured }}^{i}\right)[\mathrm{bit} / \mathrm{s} / \mathrm{Hz}]
$$

\section{B. Channel Division across Delay Spreads}

We first use emulated channels to study the impact of different channels and system parameters. For each sub-channel division factor, we evaluate the throughput improvement versus the delay spread to find the optimal division factor given a particular delay spread.

We first study the impact of diverse delay spreads on channel divisions. We set the total number of users served by an AP to be 64 and use an equal-power echo channel in this experiment (i.e., there is no attenuation in gain from the first channel tap to the second). We set the number of equal-power channel taps from 1 to 7 and the time delay between adjacent channel taps to be 50 nanoseconds, which is the inverse of the channel bandwidth. The total channel gain for different channel models is normalized to $0 \mathrm{~dB}$. For each test, we generate multi-path channel effects between each of the transmit-receive antenna pairs with a Rayleigh distribution. Then, we measure the throughput with diverse delay spread values for each channel division factor configuration listed in Table I. For each test case, we complete 1024 transmissions to obtain a reliable average result, which is shown in Figure $7 \mathrm{a}$.

We can observe that, without channel division (i.e., one subchannel), a non-zero delay spread will degrade the system throughput severely. With channel divisions, less frequency selectivity occurs within each sub-channel, achieving far better 


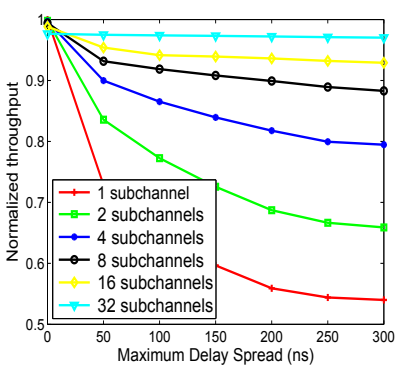

(a)

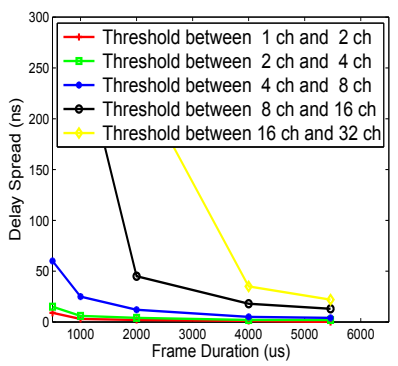

(e)

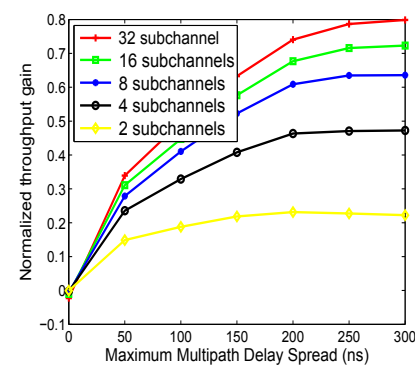

(b)

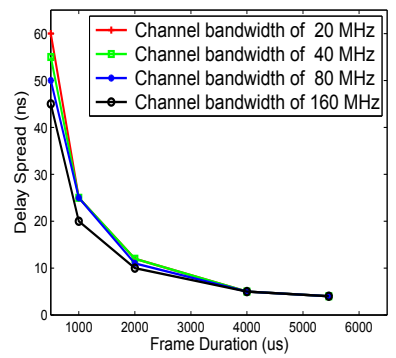

(f)

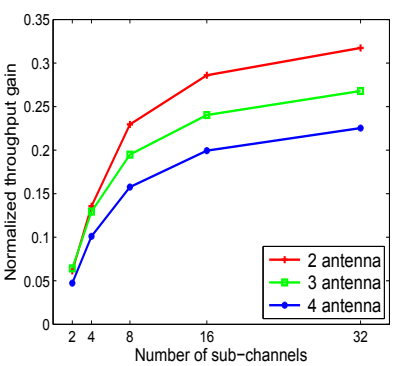

(c)

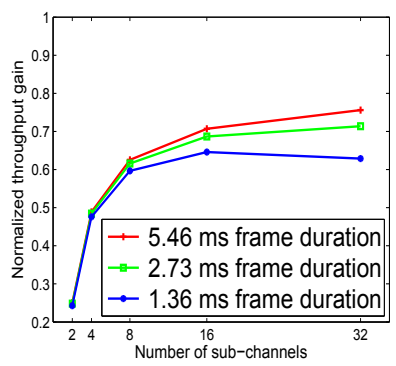

(g)

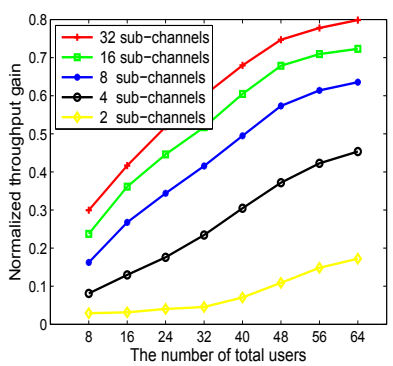

(d)

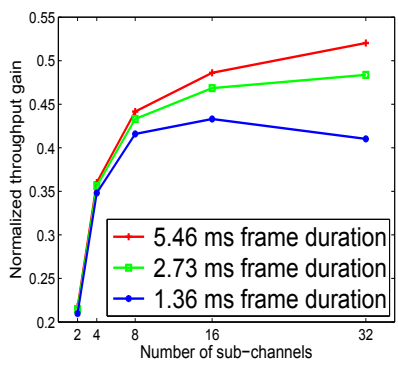

(h)

Fig. 7: Experimental results with either emulated or actual wireless channels: (a) Normalized throughput for different number of sub-channels under different channel delay spreads, (b) Normalized throughput gain for different number of sub-channels under different channel delay spreads, (c) Normalized throughput gain versus antenna numbers on the AP for 2 selected users, (d) Throughput gain versus the number of total users in the coverage of the AP, (e) Sub-channel division thresholds changes with frame duration. (f) Sub-channel division thresholds changes with channel bandwidth. (g) Normalized throughput gain in emulated IEEE TGn channel model. (h) Normalized throughput gain in an actual office environment.

channel orthogonality than that without channel divisions. Moreover, for a fixed bandwidth (e.g., $20 \mathrm{MHz}$, as in our experiments), the performance improvement of SAMU is less for a smaller delay spread due to less frequency selectivity and less of a need to divide into sub-channels. However, as bandwidth scales (e.g., $160 \mathrm{MHz}$, as in 802.11ac), even a very small delay spread (less than 50 nanoseconds) can result in severe frequency selectivity across the bandwidth. Thus, the performance of SAMU will only increase from the results reported in this paper.

To quantify the gain, we plot the improvement introduced by channel division in Figure $7 \mathrm{~b}$. We observe that, for 4 antennas and 4 selected users on each sub-channel, when the delay spread has a normalized value of 300 nanoseconds, we can achieve as much as 80 percent improvement compared to the conventional MU-MIMO scheme without channel divisions in the current 802.11ac standard. Moreover, Figure 7b shows that channel division las less of an improvement with a delay spread of 50 nanoseconds because of the reduced frequency selectivity at $20-\mathrm{MHz}$ bandwidth. However, with higher bandwidth, the trends observed here will collectively shift to the left (i.e., the effect of frequency selectivity is exacerbated even with very small delay spreads, and the use of sub-channels will only have a greater benefit for a given delay spread).

We also examined how the throughput is affected by the number of antennas on the AP. To do so, we keep the number of selected users at 2 and change the number of
AP antennas to be 2, 3, and 4. We use a 7-tap, equal-power echo channel model for this experiment. The result is shown in Figure $7 \mathrm{c}$. We observe that, as the number of antennas increase on the AP, the achieved channel division improvement decreases. This is due to channels between users tending to be more orthogonal regardless of the frequency selectivity as the number of antennas become much more than the number of selected users.

As the number of users increases, the probability of finding completely orthogonal simultaneous users increases. Hence, dividing the bands according to the level of frequency selectivity could achieve far greater throughput gains (i.e., where orthogonality in space cannot be exploited, orthogonality in frequency can be exploited). To evaluate this experimentally, we return to the default setup of 4 antennas on the AP and again use a 7-tap equal-power echo channel model. The performance for a different number of total users (with at most 4 users selected per transmission) is shown in Figure 7d. We observe that, the throughput improvement increases with the increase of the number of users. However, the increase becomes more nominal when the total user number exceeds approximately 50 users.

Furthermore, SAMU is able to adapt the number of subchannels to the delay spread and frame duration. We now evaluate the adaptation threshold for different frame durations within emulated channels and show the results in Figure 7e. We observe that as the frame duration increases, the sub- 
TABLE II: 802.11 indoor channel model

\begin{tabular}{c|c|c|c|c|c}
\hline Excess delay $(\mathrm{ns})$ & 0 & 10 & 20 & 30 & 50 \\
\hline power $(\mathrm{dB})$ & -2.6 & -3.0 & -3.5 & -3.9 & -4.5 \\
\hline Excess delay $(\mathrm{ns})$ & 80 & 110 & 140 & 180 & 230 \\
\hline power $(\mathrm{dB})$ & -5.6 & -6.9 & -8.2 & -9.8 & -11.7 \\
\hline Excess delay $(\mathrm{ns})$ & 280 & 330 & 380 & 430 & 490 \\
\hline power $(\mathrm{dB})$ & -13.9 & -16.1 & -18.3 & -20.5 & -22.9 \\
\hline \hline
\end{tabular}

channel adaptation threshold decreases, tending to use more sub-channels for the transmission. This is due to the reduction in relative overhead for the channel division with a larger frame duration, as indicated in Equation 6. Similarly, with greater bandwidth, the SA-SIG-B field will have less symbols, reducing the impact of the channel division overhead. Therefore, the adaptation threshold also decreases, as shown in Figure 7f.

\section{Diverse 802.11 Channels}

For our system evaluation, we first use a channel emulator with channel model E in 802.11 Wireless LANs TGn Models, which is for typical large indoor space with NLOS conditions (e.g., a large auditorium, conference hall, or gymnasium) [28]. The large number of users in these locations tend to improve MU-MIMO performance. The channel model profile we use is shown in Table II. We use a central frequency of $5180 \mathrm{MHz}$. We vary the frame duration to investigate the impact of the overhead introduced by the extra SA-SIG-A and SA-SIG-B symbols. The results are shown in Figure $7 \mathrm{~g}$. We observe that, with the largest frame duration setting, $5.46 \mathrm{~ms}$ specified in [7], SAMU achieves 75 percent throughput improvement over the VHT mode in 802.11ac. However, if the frame duration is smaller, the impact of the overhead introduced by the extra SA-SIG-A and SA-SIG-B symbols becomes larger, resulting in less improvement by channel division.

We also evaluated the performance gain of SAMU versus the VHT scheme in 802.11ac in the same office environment as in Section III. As depicted in Figure 7h, SAMU outperforms the existing 802.11 scheme as much as 53 percent in an actual environment. The reflections and multi-path in this office environment is not as severe as in the channel model that we use for channel emulation. If we conduct the experiments in an area with more reflections and larger delay spread, larger gains are expected. Moreover, in an environment with smaller delay spread, excessive sub-channel division will degrade the throughput due to the overhead introduced, as shown in Figure 7h. Hence, the 16 sub-channel case has better performance than the result with 32 sub-channels.

\section{CONCLUSION}

In this work, we considered frequency selectivity to optimize multi-user MIMO by considering the largest band division that can be considered frequency flat. We then chose the optimal subset of users according to their spatial orthogonality and frequency diversity. SAMU ensures user optimality in each sub-channel, and we showed that it significantly improves the multi-user performance in a network. Moreover, we proposed a selectivity-aware very high throughput (SAVHT) mode based on IEEE 802.11ac, which is an extension to the existing IEEE 802.11 standard. From our experimental results, SAMU showed as much as 80 percent throughput improvement compared to the existing systems in repeatable scenarios over the channel emulator and representative indoor environments. In the future, frequency selectivity will only increasingly dominate the behavior of $\mathrm{WiFi}$ as bandwidth scales, underscoring the importance of SAMU.

\section{ACKNOWLEDGEMENTS}

We would like to thank the anonymous reviewers for their valuable feedback. This work was supported in part by NSF grants: CNS-1318607, CNS-1456847, CNS-1150215, and CNS-1320442.

\section{REFERENCES}

[1] E. Aryafar, N. Anand, T. Salonidis, and E. W. Knightly, "Design and experimental evaluation of multi-user beamforming in wireless lans," in ACM MobiCom, 2010.

[2] T. Yoo and A. Goldsmith, "On the optimality of multiantenna broadcast scheduling using zero-forcing beamforming," Selected Areas in Communications, IEEE Journal on, vol. 24, no. 3, pp. 528-541, 2006.

[3] X. Xie and X. Zhang, "Scalable user selection for mu-mimo networks," in INFOCOM, 2014 Proceedings IEEE, 2014, pp. 808-816.

[4] H. Yu, L. Zhong, A. Sabharwal, and D. Kao, "Beamforming on mobile devices: a first study," in ACM Mobicom, 2011.

[5] Y. Du, E. Aryafar, J. Camp, and M. Chiang, "ibeam: Intelligent clientside multi-user beamforming in wireless networks," in IEEE INFOCOM, 2014.

[6] IEEE Std 802.11n, 2009.

[7] IEEE Std 802.11ac, 2014.

[8] 3GPP Long Term Evolution (LTE).

[9] K. C.-J. Lin, S. Gollakota, and D. Katabi, "Random access heterogeneous mimo networks," in ACM SIGCOMM, 2011.

[10] K. Tan, H. Liu, J. Fang, W. Wang, J. Zhang, M. Chen, and G. M. Voelker, "Sam: enabling practical spatial multiple access in wireless lan," in ACM Mobicom, 2009.

[11] S. Gollakota, S. D. Perli, and D. Katabi, "Interference alignment and cancellation," in ACM SIGCOMM, 2009.

[12] H. S. Rahul, S. Kumar, and D. Katabi, "Jmb: scaling wireless capacity with user demands," in ACM SIGCOMM, 2012.

[13] A. Bhartia, Y.-C. Chen, S. Rallapalli, and L. Qiu, "Harnessing frequency diversity in wi-fi networks," in ACM MobiCom, 2011

[14] H. K. Chung and H. L. Bertoni, "Indoor propagation characteristics at $5.2 \mathrm{ghz}$ in home and office environments," IEEE Communications and Networks, Journal of, 2002.

[15] A. Siamarou and M. Al-Nuaimi, "Wideband propagation measurements for indoor rician fading radio channels at $62.4 \mathrm{ghz}$," in Vehicular Technology Conference, 2001. VTC 2001 Spring. IEEE VTS 53rd, 2001.

[16] S.-C. Kim, H. L. Bertoni, and M. Stern, "Pulse propagation characteristics at $2.4 \mathrm{ghz}$ inside buildings," Vehicular Technology, IEEE Transactions on, 1996.

[17] "H.1. bertoni. propagation effects observed indoors." [Online]. Available: http://http://eeweb.poly.edu/faculty/bertoni/docs/06IndoorObserve.pdf

[18] T. Rappaport, S. Sun, R. Mayzus, H. Zhao, Y. Azar, K. Wang, G. Wong, J. Schulz, M. Samimi, and F. Gutierrez, "Millimeter wave mobile communications for 5g cellular: It will work!" Access, IEEE, 2013.

[19] The Multimedia over Coax Alliance (MoCA).

[20] ETSI Standard: EN 300744 V1.5.1, Digital Video Broadcasting (DVB).

[21] H. Rahul, F. Edalat, D. Katabi, and C. G. Sodini, "Frequency-aware rate adaptation and mac protocols," in ACM MobiCom, 2009.

[22] S. Yun, D. Kim, and L. Qiu, "Fine-grained spectrum adaptation in wifi networks," in ACM MobiCom, 2013.

[23] S. Singh, M. Shahbazi, K. Pelechrinis, K. Sundaresan, S. V. Krishnamurthy, and S. Addepalli, "A case for adaptive sub-carrier level power allocation in ofdma networks," in ACM MobiHoc, 2012.

[24] P. Okvist, A. Simonsson, and H. Asplund, "Lte frequency selective scheduling performance and improvements assessed by measurements," in IEEE PIMRC, 2011.

[25] A. Goldsmith, Wireless communications, 1st ed. Cambridge University Press, 2005.

[26] "The wireless open-access research platform." [Online]. Available: http://www.warpproject.org

[27] H. Viswanathan, S. Venkatesan, and H. Huang, "Downlink capacity evaluation of cellular networks with known-interference cancellation,' IEEE J.Sel. A. Commun., vol. 21, no. 5, pp. 802-811, Sep. 2006.

[28] "Tgn channel models," IEEE Standards Association, 2004. 